\title{
A Map-matching Method with a Function to Calculate Errors of Position and Direction Angle of Road Data for Car-navigation Systems
}

\author{
Toshiyuki AOKI ${ }^{\dagger}$, Mikio BANDO ${ }^{\dagger}$, Tomoaki HIRUTA ${ }^{\dagger}$, \\ Koichi KATO $^{\dagger \dagger}$, Akihiro KAWABATA ${ }^{\dagger \dagger}$, Zhixing LIU $^{\dagger \dagger}$ \\ $\dagger$ Hitachi Research Laboratory, Hitachi, Ltd. \\ 1-1, Omika-cho 7-chome, Hitachi-shi, Ibaraki, 319-1292 Japan \\ $+\quad$ Engineering Development R\&D Division, Clarion Co. ,Ltd. \\ E-mail: toshiyuki.aoki.gh@hitachi.com
}

\begin{abstract}
In car-navigation systems, car position measurement estimates the car's position and heading angle from the out-puts of a GPS receiver and sensors, and calculates the position on a link that represents a road and is recorded in a digital road map. We focus on the latter process, which is map matching. If there are large errors in the car's estimated position and heading angle as well as in link position and direction angle, map matching selects an incorrect link. We previously proposed a map-matching method that calculates the criterion from the error variances of the positions and direction angles of the links, too. However, this method must in advance measure the error variances of the positions and direction angles of the links, which are different from one area to another. This paper proposes a map-matching method with a function to calculate the error variances of the position and direction angle of the links in a digital road map and that can remove the previous measurement work. The performance of this function was evaluated, and the performance of this map-matching method was compared with that of the conventional method by using numerical computation.
\end{abstract}

\section{Introduction}

This paper proposes a map-matching method that can calculate errors of position and direction angle of road data for car-navigation systems. In car-navigation systems, car position measurement involves two processes: position estimation and map matching. The former process estimates the position, speed, heading angle, and their estimation-error covariance from the output of a Global Navigation System (GPS) receiver and sensors [1], [2]. The latter process selects the links near the car's estimated position, calculates the position and heading angle of candidate points on the links (link candidates) and selects the best link candidate, as shown in Fig. 1. Here, links represent roads and are recorded in a digital road map, link position represents the positions of all points on the link, link direc
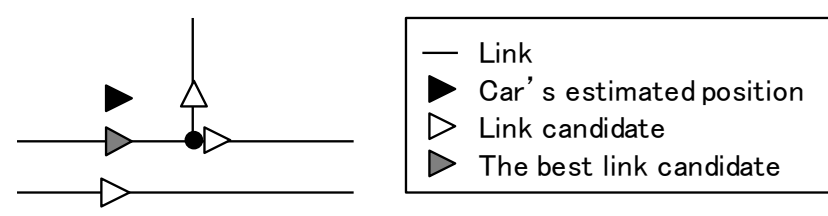

Fig. 1: Link, car's estimated position and link candidate

tion angles are the angle between the link and eastward direction, and link candidates are candidate points on the links nearest to the car's estimated position.

Many map-matching methods have been proposed and various criteria have been used to select the best link candidate [3]-[6]. For example, the criteria are calculated from the positions of a car and a link candidate (Yang et al. 2003) [3], from the positions and the heading angle of a car and a link candidate (Kim et al. 2001) [4], from the accumulative distance and accumulative angle between a car and a link candidate (Morohoshi et al. 2008) [5], and from the number of visible satellites, the link facility code, the position and heading angle of a car, their error variances, and the positions and heading angles of link candidates (Pyo et al. 2001) [6]. There are drawbacks to these methods, however. Yang's method can match the car's estimated position to an incorrect link near an intersection when the positions of a car and a link candidate contain errors, because this method does not use the heading angles of a car and a link candidate. Kim's and Morohoshi's methods do not use the error variances of the positions, heading angle, accumulative distance and accumulative angle. Consequently, when the error variances increase, large errors occur in the criteria and the methods select an incorrect link candidate. Since Pyo's method does not use the error variances of link position and direction angle, the error in the link position and direction angle sometimes causes the car's estimated position to be mismatched to a link on forks in roads, parallel roads and so on.

In light of these drawbacks, we previously proposed a method that calculates the chi-squared statistic from the distance-series data of the positions, heading angles, and their error variances of the car and the link candidates [7]. The term "distance-series data" refers to our usage of "a 
distance series" to describe a sequence of data points that are observed at constant distance intervals. Using the error variances of their positions and heading angles decreases the adverse influence on the criterion (the chi-squared statistic) even when the errors in their position and heading angle increase on forks in roads, parallel roads and so on.

However, this method must in advance measure the error variances of the positions and direction angles of the many links, which are different from one area to another. The error in the link position is a distance between a center line on a road and a link, and the error in a link direction angle is a difference between the direction angles of the center line and the link, as shown in Fig. 2. We must first plot links on aerial photo images, and then measure distances between links and center lines on roads, and differences between the direction angles of a center line and the link. The standard deviations of the errors in link position and direction angle must then be calculated from the measured distances and differences.
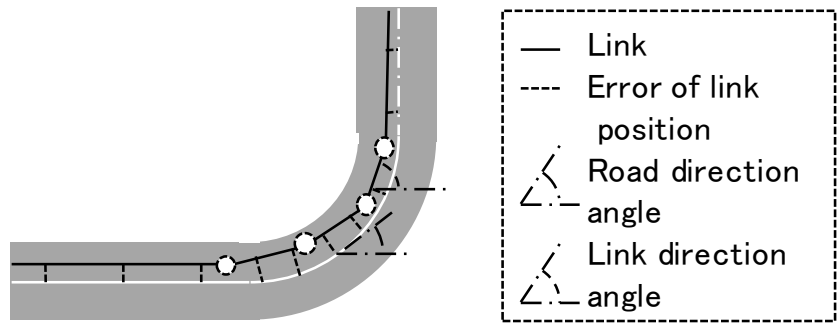

Fig. 2: Errors in link position and direction angle

This paper proposes a map-matching method that can calculate the error variances of the position and direction angle of the links in a digital road map. The car's estimated position and the link position are almost same. Therefore, Map matching can calculate the error variance of the link position by subtracting the variance of the car's estimated position from the variance from all the distance between the car's estimated position and the position of the best link candidate. As with the case of the error variance of the link position, map matching can calculate the error variance of the link direction, subtracting the variance of the car's estimated heading angle from the variance from all the difference between the car's estimated heading angle and the heading angle of the best link candidate. Therefore, this method can remove the in-advance measurement work.

This paper is organized as follows. Section 2 describes the structure of the car position measurement and the digital road map. Section 3 presents the proposed map-matching method, which uses the chi-squared statistic and calculates the error variances of the position and direction angle of the links. Section 4 details the performance of the error variance calculation and the proposed map-matching method by using numerical computation. Section 5 concludes the paper with a brief summary of key points.

\section{Structure of Car Position Measurement}

The car position measurement shown in Fig. 3 operates as follows. A GPS receiver outputs the car's position, speed, heading angle, the number of GPS signals being received, and so on. The sensors are an odometer, a gyro sensor, an accelerometer, and so on. They output the car's speed, angular velocity, and acceleration. Position estimation observes the outputs of the GPS receiver and sensors and calculates the car's estimated position, speed, heading angle and their error covariance by using a Kalman filter. Even though the system does not have any sensors, the car's estimated position, speed, heading angle and their covariance can be calculated from only the output of the GPS receiver. Map matching searches for links near the car's estimated position, calculates criteria of link candidates from the outputs of position estimation and the data in the digital road map and selects the best link candidate. It determines whether the car is running on or outside a road. If it is running on a road and if the error variances of the car's estimated position and heading angle are smaller than given values respectively, the error variances of the link position and direction angle are calculated. The error variances of the link position and direction angle that are saved in the link error database are used in the calculation of criteria of link candidates and road departure detection. If it is running on a road, the car-navigation system displays the position of the best link candidate. If it is running outside a road, the system displays the car's estimated position. We have proposed methods to select the best link candidate [7] and detect road departure [8]. This paper proposes a method to calculate the error variances of the link position and direction angle.

The digital road map contains the information about roads, which are represented by links and nodes. Nodes and links respectively represent intersections and roads between intersections. Shape points are points on the link

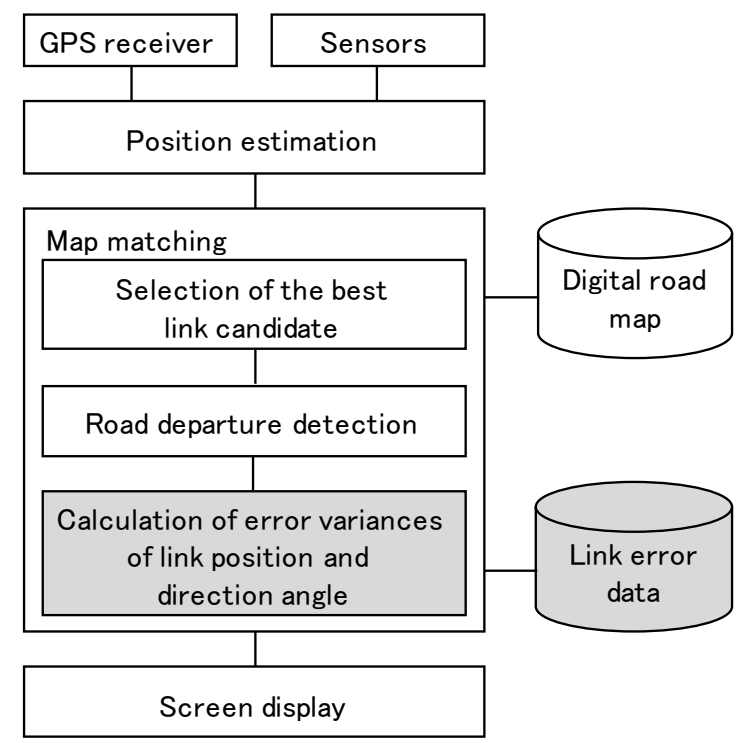

Fig. 3: Structure of car position measurement 
between the nodes. As shown in Fig. 4, the lines connecting the nodes and the shape points represent the road shape. The attributes of a link are road type, number of lanes, road width, traffic direction, speed limit, and so on. The traffic directions are two-way traffic, one-way traffic and prohibited traffic [9], [10].
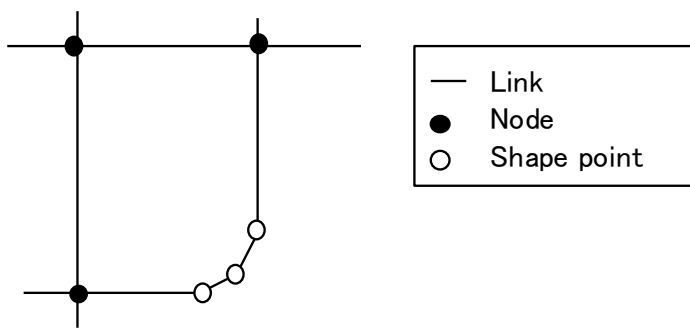

Fig. 4: Road network in digital road map

\section{Map-matching Method}

\subsection{Selection of the Best Link Candidate}

The following two conditions are satisfied when a car is traveling on a route.

Condition 1-1: The car's position and lane position are the same.

Condition 1-2: The car's heading angle and lane direction angle are the same.

We consider a criterion for indicating that Conditions 1-1 and 1-2 are satisfied. If the criterion is calculated from only the distance between the car's estimated position and the link position and the difference between the car's estimated heading angle and the link direction angle, a large error in the car's estimation or link causes map matching to select an incorrect link candidate. Therefore, the criterion is calculated from the normalized distance between the car's estimated position and the link position and the normalized difference between the car's estimated heading angle and the link direction angle. It is also difficult for map matching to select the best link candidate in parallel roads. However, because the shapes of roads to which parallel roads are connected differ, map matching can correctly select the best link candidate by calculating the criteria of the link candidates from the data of the past route (the distance-series data).

Fig. 5 shows the car's position $\left(x_{c}, y_{c}\right)$ and heading angle $\theta_{c}$, the lane position $\left(x_{r}, y_{r}\right)$ and direction $\theta_{r}$, the car's estimated position $\left(x_{e}, y_{e}\right)$ and heading angle $\theta_{e}$, the link position $\left(x_{l}, y_{l}\right)$ and direction $\theta_{l}$. The errors $\left(x_{e}, y_{e}\right)$ and $e_{\theta e}$ in the car's estimated position and heading angle, the errors $\left(e_{x l}, e_{y l}\right)$ and $e_{\theta l}$ in the link position and direction are respectively represented as follows:

$$
\begin{gathered}
{\left[\begin{array}{l}
e_{x e}(i) \\
e_{y e}(i)
\end{array}\right]=\left[\begin{array}{l}
x_{e}(i) \\
y_{e}(i)
\end{array}\right]-\left[\begin{array}{l}
x_{c}(i) \\
y_{c}(i)
\end{array}\right]} \\
e_{\theta e}(i)=\theta_{e}(i)-\theta_{c}(i)
\end{gathered}
$$

$$
\left[\begin{array}{l}
e_{l e}(i) \\
e_{l e}(i)
\end{array}\right]=\left[\begin{array}{l}
x_{l}(i) \\
y_{l}(i)
\end{array}\right]-\left[\begin{array}{l}
x_{r}(i) \\
y_{r}(i)
\end{array}\right]
$$

$$
e_{\theta l}(i)=\theta_{l}(i)-\theta_{r}(i)
$$

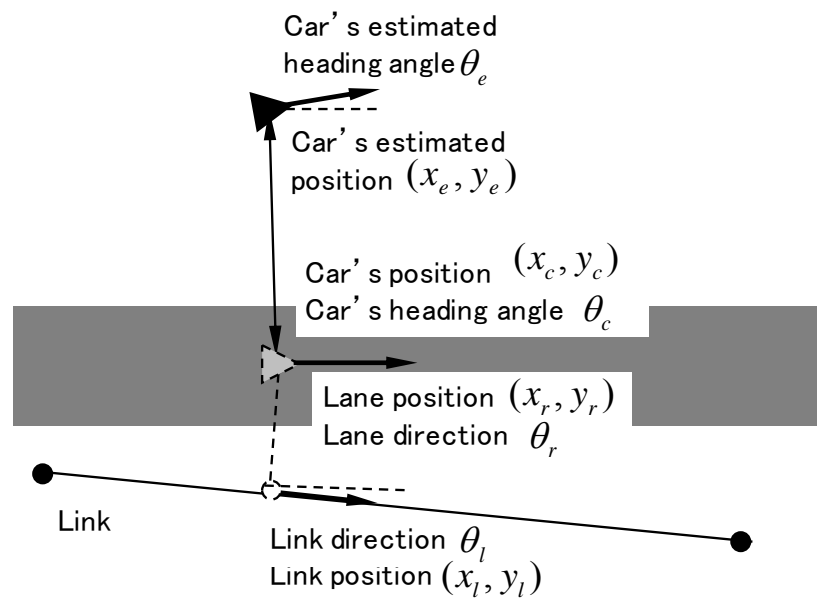

Fig. 5: Car's position and heading angle, lane position and direction in the case of one lane

It is assumed that errors in the car's estimated position and heading angle and those in the link position and direction angle are independently and normally distributed, where the error in the car's estimated position represents $\sqrt{e_{x e}^{2}+e_{y e}^{2}}$, and the error in the link position represents $\sqrt{e_{x l}^{2}+e_{y l}^{2}}$. We consider the following criterion $T$ :

$$
\begin{aligned}
& T=\sum_{i=k-n+1}^{k}\left[\begin{array}{lll}
\Delta x(i) & \Delta y(i) & \Delta \theta(i)
\end{array}\right] \Sigma_{d}^{-1}(i)\left[\begin{array}{c}
\Delta x(i) \\
\Delta y(i) \\
\Delta \theta(i)
\end{array}\right] \\
& {\left[\begin{array}{l}
\Delta x(i) \\
\Delta y(i)
\end{array}\right]=\left[\begin{array}{l}
x_{e}(i) \\
y_{e}(i)
\end{array}\right]-\left[\begin{array}{l}
x_{l r}(i) \\
y_{l r}(i)
\end{array}\right]} \\
& \Delta \theta(i)=\theta_{e}(i)-\theta_{l}(i) \\
& \boldsymbol{\Sigma}_{d}(i)=\boldsymbol{\Sigma}_{e}(i)+\boldsymbol{\Sigma}_{l}(i)
\end{aligned}
$$

$$
\boldsymbol{\Sigma}_{l}(i)=\left[\begin{array}{ccc}
\sigma_{p l}^{2} \sin ^{2} \theta_{l}(i) & 0 & 0 \\
0 & \sigma_{p l}^{2} \cos ^{2} \theta_{l}(i) & 0 \\
0 & 0 & \sigma_{\theta l}^{2}
\end{array}\right]
$$


where $\left(x_{e}, y_{e}\right), \theta_{e}$, and $\Sigma_{e}$ are respectively the car's estimated position, heading angle and their error covariance matrix, $\left(x_{l r}, y_{l r}\right)$ is a position of the point near the car's estimated position in the running region (which, as shown in Fig. 6, is an area where a car can run), $\boldsymbol{\Sigma}_{l}$ is the error covariance matrix of the link candidate position and heading angle, $\sigma_{p l}^{2}$ is the error variance of the link position, $\theta_{l}$ and $\sigma_{\theta l}^{2}$ are respectively the link direction angle and its error variance, $i$ is the number of distance-series data elements, and $n$ is the number of distance-series data elements from which the criterion is calculated.

In Fig. 6, the origin of the coordinate system $(x, y)$ is an initial estimated position, and the positive directions of the $\mathrm{x}$-axis and $\mathrm{y}$-axis are respectively eastward and northward. The white circles are points near the car's estimated position in the running region, the black circle is a node or a shape point, the black line is the link, the black triangle is the car's estimated position, and the white triangle is the link candidate, which is a point near the car's estimated position on the link.

The distance between the car's estimated position and the link candidate position is calculated as follows. The car's estimated position and its covariance are obtained from position estimation. The running region is the area between the centers of both side edges of lanes and between two lines that are perpendicular to the link and pass through the shape points or nodes. The running region is set by using the positions of the nodes and the shape points, the number of lanes, the road width, and the traffic directions that are recorded in the digital road map. The point near the car's estimated position in the running region is calculated. Then the distance between this point and the car's estimated position is calculated. This is the distance between the car's estimated position and the link candidate position. As shown in Fig. 6, in the case of two-way traffic, the number of lanes in the running region is half that in the road. In the case of one-way traffic, the number of lanes in the running region is the same as that in the road. In the case of prohibited traffic, the running region and the link candidate are not set.

The link direction is a direction parallel to the link. The link candidate heading angle is the same as the link direction angle. The car's estimated heading angle, the link candidate heading angle and the link direction angle are all angles from the $\mathrm{x}$-axis.

The error covariance of the link candidate position is presented by Eq. (9) because the error in the link candidate position distributes in a direction perpendicular to the link. The error variances of the link position and direction angle are calculated beforehand. The procedure for calculating the error variance of the link position and direction angle is described in Paragraph 4.2.

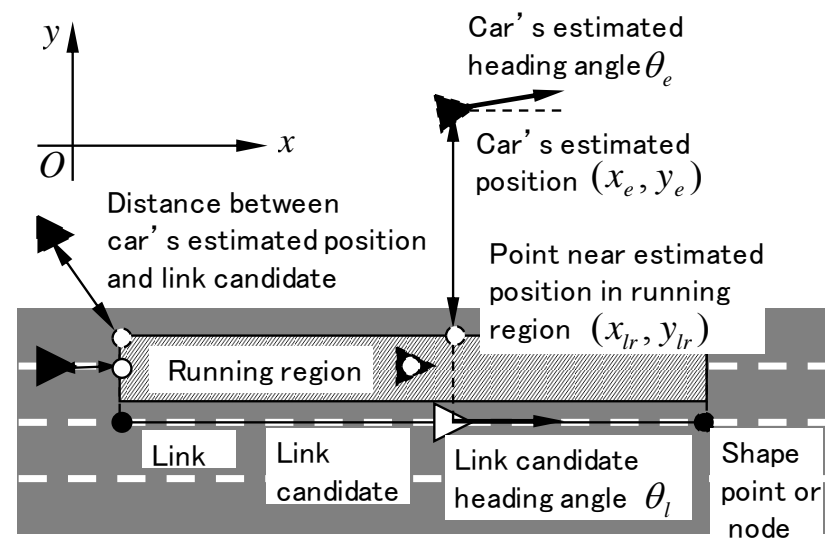

Fig. 6: Distance between car's estimated position and link candidate position

The criterion $T$ indicates the sum of the distance between the car's estimated position and the link candidate position and the difference between the car's estimated heading angle and the link candidate heading angle. Since the errors in the car's estimated position and heading angle and those in the link position and direction angle are assumed to be independently and normally distributed, where the error in the car's estimated position represents $\sqrt{e_{x e}^{2}+e_{y e}^{2}}$ and the error in the link position represents $\sqrt{e_{x l}^{2}+e_{y l}^{2}}$, it is chi-squared distributed with $2 n$ degrees of freedom. Therefore, the probability that the car is on a road is the value of the integral of the probability density function of a chi-squared distribution from the value of the criterion $T$ to infinity (Fig. 7). Fig. 7 shows the probability that the car is traveling on a route when the total number of distance-series data elements from which the criterion is calculated $(n)$ is 100 .

When the car's estimated position and heading angle and those in the link position and direction angle are assumed to be independently and normally distributed, where the error in the car's estimated position represents $\left(e_{x e}, e_{y e}\right)$ and the error in the link position represents $\left(e_{x l}, e_{y l}\right)$, the criteria $T$ is chi-squared distributed with $3 n$ degrees of freedom.

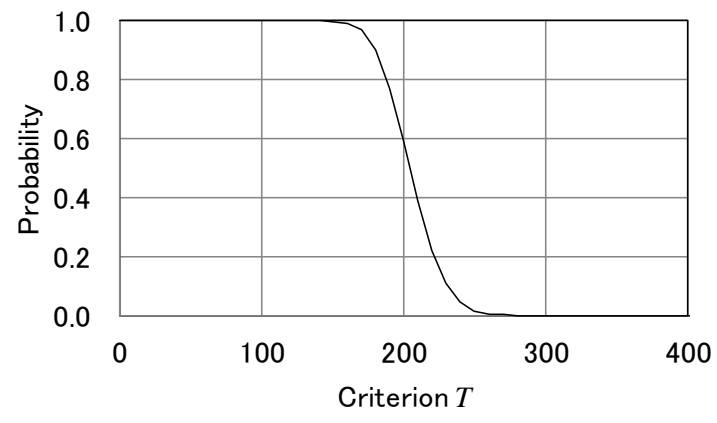

Fig. 7: Probability that the car is traveling on a route 


\subsection{Calculation of Error Variances of Link Posi- tion and Direction Angle}

This section shows the calculation of the error variance of the link position and direction angle. We define that the distance between the car's estimated position and the link position is the distance between the car's estimated position and the position of the best link candidate because the car's estimated position and the link position are almost same. The distance $l_{e l}(i)$ between the car's estimated position and the link position is represented by the following equation:

$$
\begin{aligned}
l_{c l}(i) & =\sqrt{\left(x_{e}(i)-x_{l r}(i)\right)^{2}+\left(y_{e}(i)-y_{l r}(i)\right)^{2}} \\
& =\sqrt{\left(e_{x e}(i)-e_{x l}(i)\right)^{2}+\left(e_{y e}(i)-e_{y l}(i)\right)^{2}} .
\end{aligned}
$$

The variance $\sigma_{l e l}^{2}$ of the distance $l_{e l}$ is calculated by the following equation:

$$
\begin{aligned}
\sigma_{l e l}^{2} & =\frac{1}{n_{v}} \sum_{i=1}^{n_{v}} l_{e l}^{2}(i) \\
& =\frac{1}{n_{v}} \sum_{i=1}^{n_{v}}\left(e_{x l}^{2}(i)+e_{y l}^{2}(i)+e_{x e}^{2}(i)+e_{y e}^{2}(i)\right) \\
& =\sigma_{p l}^{2}+\frac{1}{n_{v}} \sum_{i=1}^{n_{v}}\left(e_{x e}^{2}(i)+e_{y e}^{2}(i)\right) \\
& =\sigma_{p l}^{2}+\frac{1}{n_{v}} \sum_{i=1}^{n_{v}}\left(\sigma_{x e}^{2}(i)+\sigma_{y e}^{2}(i)\right)
\end{aligned}
$$

where $\sigma_{x e}^{2}$ and $\sigma_{y e}^{2}$ are respectively the error variance of the car's estimated position in eastward and northward directions.

Map matching can calculate the error variance $\sigma_{p l}^{2}$ of the link position by subtracting the variance of the car's estimated position from the variance from all the distance between the car's estimated position and the position of the best link candidate as following:

$$
\begin{aligned}
\sigma_{p l}^{2} & =\sigma_{l e l}^{2}-\frac{1}{n_{v}} \sum_{i=1}^{n_{v}}\left(e_{x e}^{2}(i)+e_{y e}^{2}(i)\right) \\
& =\frac{1}{n_{v}} \sum_{i=1}^{n_{v}} l_{e l}^{2}(i)-\frac{1}{n_{v}} \sum_{i=1}^{n_{v}}\left(e_{x e}^{2}(i)+e_{y e}^{2}(i)\right)
\end{aligned}
$$

The calculation of the error variance of the link direction angle can be obtained as with the case of the error variance of the link position. The difference $\Delta \theta(i)$ between the car's estimated heading angle and the link direction is represented by the following equation:

$$
\begin{aligned}
\Delta \theta(i) & =\theta_{e}(i)-\theta_{l}(i) \\
& =e_{\theta e}(i)-e_{\theta l}(i) .
\end{aligned}
$$

The variance $\sigma_{\Delta \theta}^{2}$ of the difference $\Delta \theta(i)$ is calculated by the following equation:

$$
\begin{aligned}
\sigma_{\Delta \theta}^{2} & =\frac{1}{n_{v}} \sum_{i=1}^{n_{v}} \Delta \theta^{2}(i) \\
& =\frac{1}{n_{v}} \sum_{i=1}^{n_{v}}\left(e_{\theta l}^{2}(i)+e_{\theta e}^{2}(i)\right) \\
& =\sigma_{\theta l}^{2}+\frac{1}{n_{v}} \sum_{i=1}^{n_{v}} e_{\theta e}^{2}(i) .
\end{aligned}
$$

Map matching can calculate the error variance $\sigma_{\theta l}^{2}$ of the link direction, subtracting the variance of the car's estimated heading angle from the variance from all the difference between the car's estimated heading angle and the heading angle of the best link candidate as following:

$$
\begin{aligned}
\sigma_{\theta l}^{2} & =\sigma_{\Delta \theta}^{2}-\frac{1}{n_{v}} \sum_{i=1}^{n_{v}} e_{\theta e}^{2}(i) \\
& =\frac{1}{n_{v}} \sum_{i=1}^{n_{v}} \Delta \theta^{2}(i)-\frac{1}{n_{v}} \sum_{i=1}^{n_{v}} e_{\theta e}^{2}(i) \\
& =\frac{1}{n_{v}} \sum_{i=1}^{n_{v}} \Delta \theta^{2}(i)-\frac{1}{n_{v}} \sum_{i=1}^{n_{v}} \sigma_{\theta e}^{2}(i)
\end{aligned}
$$

where $\sigma_{\theta e}^{2}$ is the error variance of the car's estimated heading angle.

In other to accurately calculate the error variance $\sigma_{p l}^{2}$ of the link position, map matching calculates it when Conditions 3-1, 3-2 and 3-3 are satisfied.

Condition 3-1: Map matching determines that the car is running on a road.

Condition 3-2: Horizontal Dilution of Precision (HDOP) is smaller than a given value, where HDOP shows geometric arrangement of GPS satellites. The smaller the value of HDOP the more accurate the position output in a GPS receiver.

Condition 3-3: Error variance of the car's estimated position is smaller than a given value.

Map matching calculates the error variance of the link direction as with the case of the error variance of the link position when Conditions 3-1, 3-2 and 3-4 are satisfied.

Condition 3-4: Error variance of the car's estimated heading angle is smaller than a given value.

In the future, we will consider a method for when a road has two or more lanes. 


\section{Performance Evaluation by Using Numer- ical Computation}

\subsection{Performance Evaluation for Link Error Calculation}

This section evaluates the performance of the method for calculating error variances of link position and direction angle by using numerical computation. We considered a simple model that satisfies the following conditions.

Condition 3-1: The road is straight.

Condition 3-2: The road has a traffic lane.

Condition 3-3: The links are 50 meters long.

Condition 3-4: The position errors of shape points follow a normal distribution with mean 0 and variance 2.25 square meters.

Condition 3-5: The car is running on a road.

Condition 3-6: The error variances of the car's estimated position and heading angle are the same as given values (4 square meters and 1 square degree) in Conditions 2-3 and $2-4$

We made links that satisfy Conditions 3-1 to 3-4. Figs. 8 and 9 show the error of the link position and direction angle. We implemented a numerical computation of calculation of error variances of link position and direction angle shown in Section 3.2 and obtained the results shown in Table 1. The proposed method calculated the error standard deviations of link position and direction angle with $2.0 \%$ and $3.1 \%$ accuracy, respectively.

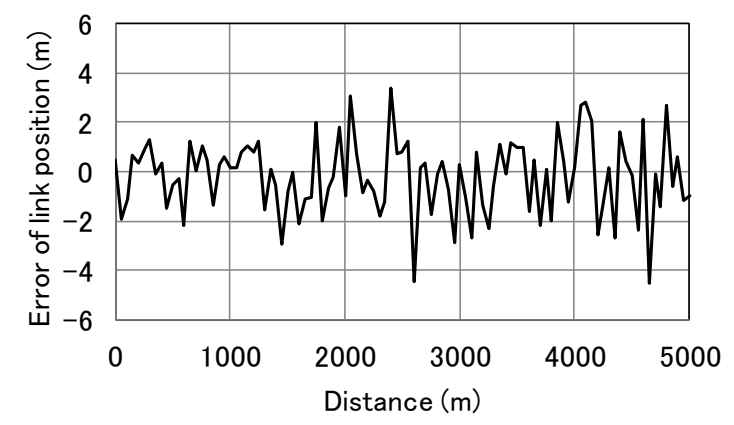

Fig. 8: Error of link position

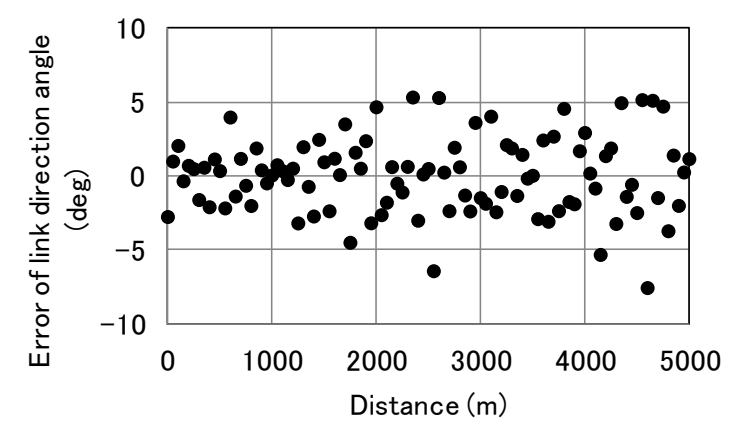

Fig. 9: Error of link direction angle
Table 1: Results in a numerical computation

\begin{tabular}{|l|c|c|}
\hline \multicolumn{1}{|c|}{ Item } & True value & Calculated value \\
\hline $\begin{array}{l}\text { Error variance of link } \\
\text { position }\end{array}$ & $5.75 \mathrm{~m}^{2}$ & $5.53 \mathrm{~m}^{2}$ \\
\hline $\begin{array}{l}\text { Error variance of link } \\
\text { direction angle }\end{array}$ & $6.62 \mathrm{deg}^{2}$ & $7.06 \mathrm{deg}^{2}$ \\
\hline
\end{tabular}

\subsection{Performance Evaluation for Map Matching}

We compared the proposed method with a conventional method. Such methods (for example Yang's method, Morohashi's method and Pyo's method) do not use the errors in the link positions or direction angles. Therefore, we the proposed method compared with the conventional method that calculated the criterion $T_{t r}$ :

$$
T_{t r}=\sum_{i=k-n+1}^{k}\left[\begin{array}{lll}
\Delta x(i) & \Delta y(i) & \Delta \theta(i)
\end{array}\right] \Sigma_{e}^{-1}(i)\left[\begin{array}{c}
\Delta x(i) \\
\Delta y(i) \\
\Delta \theta(i)
\end{array}\right]
$$

This conventional method calculates the criterion without using the error variances of the link positions and direction angles.

Almost all links of forks in roads have errors in link positions and direction angles as shown in Fig. 10. Some links of parallel roads have errors in link positions and direction angles as shown in Fig. 11. Therefore, we compared the performances of the proposed and the conventional methods.

We considered the conditions in which the links of the road on which the car is running have errors, other links have no errors, and the values of other parameters are shown Table 2. The values, which were calculated in the performance evaluation for link error calculation as shown Section 4.1, are set to the error variances of link position and direction angle. We compared map-matching performances of the conventional and proposed methods by processing the map-matching algorithm.

In the case shown in Fig. 10 in which the error in the link direction angle is 6.8 degrees on the forks in roads where the angle between roads is 5.7 degrees, the conventional method selects the incorrect link candidate, but the proposed method selects the correct link candidate.

In the case shown in Fig. 11 in which the error in the link angle is 5 degrees, the conventional method selects the incorrect link candidate, but the proposed method selects the correct link candidate. Consequently, it is demonstrated that the proposed map-matching method outperforms the conventional method when links of forks in roads and parallel roads have errors in the link positions and direction angles. 


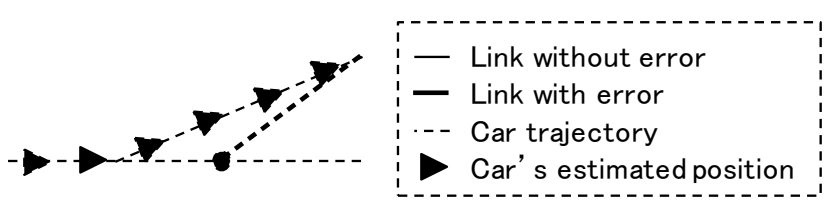

Fig. 10: Links with error on forks in roads

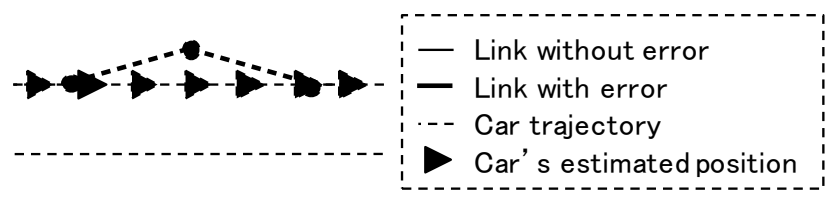

Fig. 11: Links with error on parallel roads

Table 2: Parameters for processing map matching

\begin{tabular}{|l|c|}
\hline \multicolumn{1}{|c|}{ Parameter } & Value \\
\hline Error variance of car's estimated position & $9 \mathrm{~m}^{2}$ \\
\hline $\begin{array}{l}\text { Error variance of car's estimated heading } \\
\text { angle }\end{array}$ & $1 \mathrm{deg}^{2}$ \\
\hline Error variance of link position & $5.75 \mathrm{~m}^{2}$ \\
\hline Error variance of link direction angle & $7.06 \mathrm{deg}^{2}$ \\
\hline
\end{tabular}

\section{Conclusions}

We proposed a map-matching method that can calculate the errors of link position and direction angle for car-navigation systems. This method measures the errors in the link position and direction angle and calculates their variances, if map matching determines that the car is running on a road and if the car's estimated position and heading angle are accurate. This method can remove the in-advance work in which people measure the errors of link position and direction angle using aerial photo images. This method calculated the error standard deviations of link position and direction angle with $2.0 \%$ and $3.1 \%$ accuracy, respectively, under conditions in which a road is straight and has a traffic lane. It was demonstrated that the proposed map-matching method selected correct link candidates while the conventional method did not when the links of the road on which the car is running have the errors, and other links do not have the error on forks in roads and parallel roads. In the future, we will consider this method for roads that have two or more lanes and will show that it accurately calculates the error variances of link position and direction angle.

\section{References}

[1] M. Bando, Y. Kawamata, and T. Aoki: Dynamic Sensor Bias Correction for Attitude Estimation using Unscented Kalman Filter in Autonomous Vehicle, Proceedings of the 42th ISCIE International Symposium on Stochastic Systems Theory and Its Applications, pp. 33-39, Okayama, November 2010.

[2] M. Bando, Y. Kawamata, T. Aoki, and K. Tanaka: The Study of Localization and Attitude Estimation for Autonomous Medium Speed Vehicle in an Outdoor Location, Proceedings of SICE 10th Annual Conference on Control Systems, 161-1-4, Kumamoto,
March 2010.

[3] D. Yang, B. Cai, and Y. Yuan: An Improved Map-Matching Algorithm Used in Vehicle Navigation System, IEEE Proceeding on Intelligent Transportation Systems, pp. 1246-1250, 2003.

[4] S. Kim, and J. Kim: Adaptive Fuzzy-Network-Based C-Measure Map-Matching Algorithm for Car Navigation System, IEEE Transactions on Industrial Electronics, Vol. 48, No. 2, April 2001.

[5] H. Morohashi, K. Abe, Y. Tsukamoto, K. Uehara, and H. Seino: A Map Matching Algorithm using Vehicle Existence Coefficient, The 25th Sensing Forum, pp. 268-271, Saga, November 2008.

[6] J. Pyo, D. Shin, and T. Sung: Development of a Map Matching method using the multiple hypothesis technique, 2001 IEEE Intelligent Transportation Systems Conference Proceedings, pp. 23-27, Oakland, August 2001.

[7] T. Aoki, M. Bando, T. Hiruta, K. Kato, A. Kawabata, and Z. Liu: A Map-matching Method Using a Chi-squared Statistic for Car-navigation Systems, Proceedings of the 44th ISCIE International Symposium on Stochastic Systems Theory and Its Applications, pp. 87-93, Tokyo, November 2012.

[8] T. Aoki, M. Bando, T. Hiruta, K. Kato, A. Kawabata, and Z. Liu: Road Departure Detection Using State Diagrams and Statistical Hypothesis Tests in Map Matching, Transactions of ISCIE, Vol. 26, No. 2, pp. 53-61, February 2013.

[9] K. Li, H. Tan, J. Misener and K. Hedrick: Digital Map as a Virtual Sensor - Dynamic Road Curve Re-construction for a Curve Speed Assistant, Vehicle System Dynamics, Vol. 46, No. 12, pp. 1141-1158, December 2008.

[10] J. Magenheim: Developing the AGORA road map ANDIL: AGORA Network against Digital Divide by means of Information Literacy, Joint Open and Working IFIP Conference ICT and Learning for the Net Generation, Kuala Lumpur, Malaysia, July 2008. 